\title{
Conhecimento e reflexões sobre estomias de eliminação: uma abordagem em grupo com enfermeiras
}

\author{
Knowledge and reflections on elimination stomas: a group approach with nurses \\ Conocimiento y reflexiones sobre estomas de eliminación: un enfoque de grupo con enfermeras
}

\begin{abstract}
RESUMO
Objetivo: Relatar os saberes e vivências de enfermeiras sobre estomias de eliminação durante a participação em uma educação permanente em saúde. Método: Estudo descritivo, com abordagem qualitativa, de caráter analítico e interpretativo, desenvolvido com enfermeiras. Foi construído um grupo focal para a realização da atividade de educação permanente. Resultados: A partir da análise, surgiram três categorias temáticas: experiências com estomias de eliminação, desafios que envolvem o cuidado da pessoa com estomia de eliminação e percepções sobre o grupo focal. Conclusão: A educação permanente favoreceu o aprofundamento do conhecimento e o amadurecimento profissional das participantes, promoveu discussões e debates que podem melhorar a prática clínica e reflexões quanto ao papel do enfermeiro sobre a assistência à pessoa com estomia de eliminação.

Descritores: Enfermagem; Educação Continuada; Estomia; Cuidados de enfermagem.
\end{abstract}

\section{ABSTRACT}

Purpose: To provide a report on nurses' knowledge and experiences about elimination stoma during their participation in permanent health education. Methods: An analytical, interpretative, and descriptive study with a qualitative approach developed with six nurses. A focus group was built to carry out the permanent education program. Results: Three thematic categories emerged from the analysis: a) experiences with elimination stoma, b) challenges involving the care of the person with a stoma; and c) perceptions on the focus group. Conclusion: The permanent education program favored knowledge development and the professional growth of the participants; it also promoted discussions and debates that can improve clinical practice, and reflections on the role of nurses in the care of people with elimination stoma.

Descriptors: Nursing; Continuing Education; Stoma; Nursing Care.

\section{RESUMEN}

Objetivo: Reportar los conocimientos y experiencias de las enfermeras sobre las estomas de eliminación durante la participación en una educación permanente en salud. Método: Estudio descriptivo, con abordaje cualitativo, de caráter analítico e interpretativo, desarrollado con enfermeras. Se construyó un grupo focal para realizar la actividad de educación permanente. Resultados: Del análisis surgieron tres categorías temáticas: experiencias con estoma de eliminación, desafíos relacionados con el cuidado de la persona con estoma de eliminación y percepciones sobre el grupo focal. Conclusión: La educación permanente favoreció la profundización en el conocimiento y la maduración profesional de las participantes, promovió discusiones y debates que pueden mejorar la práctica clínica, y reflexiones sobre el papel de las enfermeras en el cuidado de las personas con estoma de eliminación.

Descriptores: Enfermería; Educación Continua; Estoma; Cuidado de Enfermería.

\section{Priscilla Nicácio da Silva ${ }^{1}$ \\ (1) $\underline{0000-0003-3489-552 X}$ \\ Izabella Chrystina Rocha \\ (1) $\underline{0000-0002-7719-6588}$}

Diovana Marielle Alves Bueno²

(1) 0000-0003-0855-3139

Mariane de Moraes Rezende da Silva ${ }^{2}$ (D) $\underline{0000-0001-8898-0522}$

Satie Katagiri ${ }^{2}$

$\underline{0000-0002-7812-2396}$

Ivone Kamada ${ }^{1}$

0000-0003-2569-8727

${ }^{1}$ Universidade de Brasília.

${ }^{2}$ Universidade Federal de Mato Grosso.

Autor correspondente:

Priscilla Nicácio da Silva

E-mail: prisca.nicacio@gmail.com

\section{Como citar este artigo:}

Silva PN, Rocha IC, Bueno DMA, et al. Estomias de eliminação: uma abordagem em grupo com enfermeiras. Revista de Enfermagem do Centro-Oeste Mineiro. 2021;11:e4135. [Access__]; Available in:__. DOI: http://doi.org/10.19175/recom.v11i0.4135 


\section{INTRODUÇÃO}

A construção de uma estomia de eliminação ocorre, quando, por diversas causas, há impossibilidade de eliminação fisiológica de dejetos e flatos. É definida pela realização de uma abertura cirúrgica entre um órgão oco e o meio externo, com a finalidade de eliminar os dejetos e flatos, em equipamentos coletores, de forma temporária ou definitiva, sendo classificada em urostomia, ileostomia e colostomia ${ }^{(1-2)}$. $\mathrm{Na}$ prestação do cuidado a esses pacientes, o enfermeiro é um dos primeiros integrantes da equipe multidisciplinar a desenvolver ações em saúde, devendo possuir qualidade técnica e científica na dispensação do cuidado, prevenir e detectar precocemente complicações e promover ações de educação em saúde ${ }^{(3-4)}$.

A existência de complicações, após confecção da estomia, possui relação com o inadequado manejo terapêutico, em alguma fase de intervenção e, com a inexistência ou oferta insuficiente de orientações, durante o período perioperatório e no pós-alta. As principais complicações em estomias de eliminação relacionam-se à pele periestomia, como lesões e dermatite e ao estoma, como sangramentos, extravasamento de resíduos, hérnias, infecções, estenose, prolapso, retração e necrose ${ }^{(5)}$.

A pessoa com estomia necessita que a assistência em saúde possua envolvimento interprofissional no manejo seguro de técnicas, educação em saúde, incentivo ao autocuidado e avaliação continuada em todo o período que abrange a estomização. Nesse percurso, o enfermeiro exerce importante papel na realização de avaliações e intervenções, além de atuar como facilitador do processo adaptativo da pessoa com estoma e dos cuidadores. Para isso, é indispensável a existência de domínio clínico terapêutico para a dispensação de técnicas seguras e orientações oportunas $^{(4)}$

A familiarização com intervenções clínicas e terapêuticas a pessoas com estomias de eliminação deve ser iniciada, durante a formação, na graduação em enfermagem e perdurar por todo o exercício profissional dos enfermeiros. Entretanto adversidades podem surgir, durante esse percurso, apresentando-se como obstáculos que dificultam o processo de ensino e aprendizagem satisfatório, sendo necessária a constante atualização e aprimoramento dos saberes no período de exercício profissional|(3). Nesse contexto, a Educação Permanente em Saúde
(EPS) apresenta-se como alternativa viável e eficiente, pois consiste em estratégia de inovação e organização do processo de trabalho em saúde, que utiliza a educação em serviço, para o fortalecimento e desenvolvimento de competências aos profissionais de saúde, com inserção de atualizações ao processo de cuidado ${ }^{(6-}$ 7).

Assim, a realização de capacitação dos profissionais envolvidos na assistência a pessoas com estomias deve ser uma prática frequente, visto que contribui para a qualidade da assistência, assegura a tomada de decisões e sistematiza as ações em saúde ${ }^{(8)}$.

A partir da compreensão do papel do enfermeiro quanto ao cuidado a pessoas com estomias de eliminação, justificou-se este estudo pelo seguinte questionamento: quais os conhecimentos e experiências vivenciadas por enfermeiros atuantes na atenção secundária sobre estomias de eliminação? Nessa perspectiva, objetiva-se com este estudo relatar os saberes e vivências de enfermeiras sobre estomias de eliminação durante a participação em uma educação permanente em saúde.

\section{MÉTODO}

Estudo descritivo e transversal com abordagem qualitativa, de caráter analítico e interpretativo, desenvolvido em um município localizado no interior do Estado de Mato Grosso, Brasil, que utilizou um grupo focal para o desenvolvimento de atividade de educação permanente.

Os critérios de inclusão dos participantes na pesquisa foram ter graduação em enfermagem, trabalhar há pelo menos seis meses na rede secundária em saúde e participar de todas as etapas propostas para o desenvolvimento do estudo. A pesquisa teve início em dezembro de 2019 e foi finalizada em fevereiro de 2020. Ocorreu, por meio da realização de três encontros, sendo que cada um envolveu uma etapa da coleta de dados e teve como local de execução a sala de reuniões de um hospital público municipal.

O hospital onde ocorreram as fases da pesquisa presta cuidados especializados em saúde, em diversas áreas, atende pacientes provenientes de 16 municípios vizinhos e configura-se no local de trabalho dos enfermeiros convidados a participar da pesquisa.

A primeira etapa do estudo consistiu na eleição, convite e concordância de 30 enfermeiros, 
sendo dois homens e 28 mulheres, atuantes na rede secundária em saúde, a participar de uma educação permanente em saúde sobre estomias de eliminação. Após o aceite, foi realizada uma entrevista individual, gravada em áudio, guiada por um instrumento semiestruturado, que investigou o perfil sociodemográfico e o conhecimento em relação à temática estomias de eliminação. 0 instrumento investigou se o enfermeiro sabia o que era uma estomia de eliminação, se já havia prestado assistência a pacientes com esses estomas, quais cuidados prestou ou prestava a esses pacientes e quais dificuldades enfrentou na dispensação desses cuidados.

Em seguida, foi organizado um roteiro estruturado, para a abordagem em grupo, baseado nos conhecimentos identificados, após análise das entrevistas e evidências científicas, que serviu de guia para a composição de um grupo focal, que teve por objetivo discutir e aprofundar os conceitos relacionados a estomias de eliminação na prática clínica dos enfermeiros. O grupo focal constituiu-se de uma técnica de produção de dados que se fundamentou na formação de grupos com características comuns, conduzidos por um facilitador, que manteve a dinâmica de discussões e centralidade no tema investigado. Permitiu o compartilhamento de experiências, saberes, ideias e sentimentos, possibilitando o surgimento de reflexões, incorporação de novos conceitos e aprimoramento de conhecimento já adquirido ${ }^{(9)}$.

Foram realizados dois encontros em grupo com início às 20 horas, após a troca de plantão de trabalho, envolvendo pessoas do plantão diurno e noturno, com duração média de 70 minutos e gravados em áudio e vídeo integralmente. $\mathrm{Na}$ primeira sessão, foram apresentados, por meio de imagens e diálogo guiado pelo roteiro, o conceito de estomia de eliminação e as complicações prevalentes na pessoa com esse tipo de estoma e, logo após essa explanação, foram iniciadas as discussões em grupo. No segundo encontro, foi apresentado e discutido o uso adequado de equipamentos coletores e adjuvantes disponíveis para a prevenção e tratamento de complicações em estomias, sendo utilizado um manequim de baixa fidelidade para a demonstração técnica. Nessa etapa, investigaram-se as percepções dos participantes sobre a educação permanente realizada.

A análise inicial dos achados deu-se, por meio da transcrição e interpretação das entrevistas da primeira etapa, que delineou os saberes dos enfermeiros sobre estomias de eliminação. Posterior à realização dos encontros do grupo focal, efetuou-se a análise dos dados produzidos nessas reuniões, que ocorreu, logo depois do exame acurado das imagens em vídeo e som de voz, de forma a elencar os pontos-chave das discussões. Os registros foram ordenados, transcritos e codificados de forma a abordar os dados qualitativos de maneira flexível e independente ${ }^{(10)}$.

Para o adequado registro participativo das enfermeiras, foram identificadas com a letra E, seguida de algarismo arábico que representa a ordem de participação nas primeiras falas do grupo. Todas as participantes assinaram Termo de Consentimento Livre e Esclarecido e Termo de Imagem e Som de Voz. O estudo foi submetido ao Comitê de Ética em Pesquisa e aprovado sob o número de parecer 3.675.794 e Certificado de Apresentação de Apreciação Ética 23373119.7.0000.5587.

\section{RESULTADOS}

Participaram integralmente da pesquisa seis sujeitos do sexo feminino, quatro atuantes na assistência direta ao paciente e duas responsáveis técnicas de enfermagem. A média de idade entre as participantes foi de 36 anos, sendo três participantes casadas, duas solteiras e uma divorciada. No quesito filhos, quatro possuíam pelo menos um filho. Os setores de trabalho das enfermeiras, nas unidades de saúde, foram centro cirúrgico (n1), unidade de terapia intensiva (n1) internação adulto $(n 1)$, internação pediátrica $(n 1)$ e responsabilidade técnica e gestão de equipe (n2), com regime de trabalho de 12 horas de plantão por 36 horas de folga, totalizando 40 horas semanais de trabalho. As participantes também foram inquiridas quanto ao exercício profissional, em outra instituição e averiguou-se que duas participantes possuíam outro vínculo empregatício, uma em rede pública e uma em rede particular. Todas as entrevistadas relataram realizar atualização profissional com frequência de pelo menos um ano em temas diversificados e afirmaram nunca ter participado de nenhuma ação de educação permanente referente a estomias de eliminação.

Quando interpeladas na primeira fase do estudo a respeito dos seus saberes, em relação a estomias de eliminação, todas as entrevistadas afirmaram saber o que era um estoma de eliminação e ainda prestar cuidados a pessoas com 
esse tipo de estomia, no entanto não conseguiram discorrer em suas falas o conceito apropriado ao tema, pois relacionaram os estomas de eliminação apenas à construção de uma colostomia. A partir dessa informação, optou-se por iniciar o grupo focal pela definição de estomia de eliminação e descrição das complicações mais prevalentes, no contexto da assistência à pessoa com esse tipo de estomia, o que auxiliou no corpus da pesquisa e permitiu a elaboração das categorias temáticas.

Após análise acurada e categorização dos relatos, surgiram os seguintes eixos temáticos: experiências com estomias de eliminação; desafios que envolvem o cuidado da pessoa com estomia de eliminação e percepções sobre o grupo focal.

\section{Experiências com estomias de eliminação}

Na primeira reunião do grupo, após a apresentação dos conceitos e complicações em estomias, as participantes foram incentivadas a comentar suas experiências sobre $o$ atendimento a esses pacientes. Nesse domínio, surgiram diálogos que revelaram o entendimento a respeito do conceito de estomia de eliminação e explanações sobre a prestação do cuidado aos pacientes.

"Eu não tinha certeza do conceito, sabia das intestinais. [...] Na maioria das vezes, a gente só faz a troca do equipamento mesmo. A avaliação deixa a desejar" (E1). "Olha, esse tema não é muito abordado, pelo menos eu nunca tinha visto. Quando a gente vê esses pacientes, fazemos o que dá para fazer, na maioria das vezes, é só a troca da bolsa. Eu não pensava muito na parte psicológica ou no que é usar a bolsa" (E2). "Eu não sabia de muita coisa que falou aqui [...] de verdade, eu só pensava na troca da bolsa, eu não sabia muito o que fazer para melhorar para o paciente" (E3).

As partícipes também discorreram sobre o perfil dos pacientes atendidos por elas nas unidades de saúde à qual prestavam assistência. Os relatos revelaram que, geralmente, os pacientes com estomia atendidos por elas são do sexo masculino, possuem estomias intestinais temporárias, sendo a causa mais frequente, para a confecção do estoma, a obstrução intestinal e o trauma abdominal.

"Eu tenho uma quantidade razoável de pacientes assim, muitos por causa de alguma obstrução intestinal, daí tem que fazer a cirurgia. A maioria dos pacientes são homens" (E2). "Eu recebo paciente que faz estomia por trauma, ferimento por arma ou por obstrução. A idade é misturada e realmente a maioria é homem" (E4). "Do tempo que trabalho aqui a maioria dos pacientes são homens com colostomia temporária" (E5).

Quando instigadas sobre as complicações em estomias de eliminação que já observaram em sua prática clínica, percebeu-se que as enfermeiras não conheciam algumas das complicações apresentadas ao grupo e apontaram a dermatite como a complicação mais prevalente em seus pacientes. As complicações em estomias abordadas com as participantes, na primeira sessão do grupo, foram a dermatite, estenose, hérnia, prolapso e retração.

"Geralmente a complicação mais comum é dermatite mesmo. Às vezes, tem paciente com estenose. Essas outras eu não vi ainda" (E1). "Olha, o que é muito comum aqui é a dermatite e já vi paciente com retração. Essas outras eu nunca vi, na verdade, nem sabia de algumas delas [risos]" (E2). "Aqui a gente vê muita dermatite [...]porque cada vez que troca a bolsa lesiona a pele" (E5).

\section{Desafios que envolvem o cuidado da pessoa com estomia de eliminação}

Nessa categoria, foram agrupados os relatos que apresentaram os desafios enfrentados pelas participantes na realização da assistência a pessoas com estomia. A princípio, foram apontados, como desafios, o conhecimento profissional sobre estomias de eliminação, indicado por vezes como diminuto e a inexistência de materiais e equipamentos disponíveis.

"Eu acho que falta mesmo é o domínio do assunto. É saber o que fazer. Tem muita coisa que nós não sabíamos e a gente atende pacientes assim aqui" (E3). "O que atrapalha o cuidado adequado é o conhecimento mesmo. Se tem conhecimento você faz" (E5). "Eu concordo que o conhecimento é fundamental, mas ter material, equipamentos e estrutura para fazer o cuidado é indispensável também" (E2).

Após aprofundamento nas discussões sobre esses desafios, também foram apontadas a rotina de trabalho e a falta de diálogo entre a equipe de saúde, como fatores que promovem comodismo e, consequentemente, dificuldades para a prestação do cuidado integral.

"Eu vejo muitos fatores que fazem o atendimento ficar, às vezes, insuficiente $e$ 
mecânico. A rotina do serviço, porque a gente tem pacientes graves e muito diferentes, os registros da enfermagem, que, às vezes, não estão bons e a conversa entre a equipe" (E1). "Olha, eu acho que a rotina é cansativa e faz a gente não correr atrás de atualizar. Outra coisa é o trabalho entre a equipe que, às vezes, é difícil" (E2).

Em meio a discussões sobre os obstáculos que permeiam a assistência à pessoa com estomia de eliminação, percebeu-se uniformidade de opiniões entre as participantes sobre os desafios elencados, com interação complementar e concordância entre os diálogos.

\section{Percepções sobre o grupo focal}

No segundo encontro do grupo, discutiu-se sobre os equipamentos e adjuvantes disponíveis para a prevenção e terapêutica de complicações em estomias de eliminação. As falas expuseram pouca familiaridade das participantes sobre a existência de boa parte dos equipamentos e adjuvantes expostos, o que norteou as discussões para a relevância e percepções trazidas pelo grupo à prática clínica das participantes.

"Eu achei bom, trouxe uma noção e um entendimento que eu não tinha. Só acho que faltou a parte prática, uma simulação por exemplo" (E1). "Eu gostei muito. Nossa, tem muita coisa que foi abordada aqui que eu não sabia. Vai fazer muita diferença quando eu atender um paciente assim" (E2). "Achei ótimo. Tem que ter mais momentos assim, porque a gente aprende muito" (E5).

A eleição da temática abordada no grupo foi elogiada e incentivada pelas enfermeiras, o que assinalou a importância em se trabalhar com o assunto ao estabelecimento e execução de uma boa conduta de enfermagem no cuidado com pessoas com essas estomias.

"Eu achei o tema ótimo, principalmente, porque é uma vivência até comum aqui" (E3). "Acho que a escolha do tema e de fazer isso em grupo foi boa para fixar o conhecimento e fazer a gente refletir sobre isso, porque melhora a assistência" (E5). "Eu achei muito bom, porque nunca tinha participado de um grupo assim e a gente ficou livre para falar e perguntar. Acho que seria bom para todos se isso fosse feito mais vezes" (E4).

No que tange às opiniões sobre a educação permanente (EPS) em saúde ofertada e método proposto, as participantes relataram sentir confiança e liberdade, para se expressarem e analisaram benefícios da EPS, para a qualidade do trabalho de enfermagem, segurança na realização da assistência e compreensão do papel do enfermeiro nesse processo de cuidado.

\section{DISCUSSÃO}

No tocante ao perfil de gênero, houve semelhança com outros estudos realizados na atenção secundária em saúde quanto à predominância de mulheres ${ }^{(11-12)}$. Esse achado é uma característica da profissão de enfermagem, ainda identificada como uma profissão com intensa força de trabalho feminino ${ }^{(12)}$.

A pesquisa mostrou limitações quanto ao nível de conhecimento das entrevistadas sobre o conceito de estomias de eliminação e complicações prevalentes nas pessoas com esses estomas. Esse resultado corrobora com outra pesquisa que verificou o conhecimento do profissional de enfermagem no cuidado a pessoas com estomias e apresentou achados que evidenciaram fragilidades no nível de conhecimento quanto à classificação das estomias, eliminações fecais e equipamentos coletores ${ }^{(4)}$. Destaca-se a importância do enfermeiro no cuidado à essa população, sendo fundamental a qualificação na execução de habilidades técnicas e conhecimento científico e situacional.

Quanto às complicações evidenciadas na prática dos enfermeiros com pessoas estomizadas, a dermatite foi apontada como principal complicação, o que se assemelhou a outros estudos $^{(2,13)}$. As complicações em pessoas com estomias de eliminação ocorrem em 30\% dos pacientes, dividindo-se entre mediatas, imediatas e tardias. A dermatite é caracterizada como uma complicação evitável, geralmente relacionada à umidade, fricção, lesão química por contato, reação alérgica ou trauma mecânico ${ }^{(14-15)}$. Para evitar essa complicação ou mesmo controlá-la, salienta-se a importância do corte ajustado da placa adesiva, o uso de adjuvantes barreira e esvaziamento do equipamento coletor com $1 / 3$ de resíduo ${ }^{(2,16)}$, informações e ações que devem ser fornecidas pela equipe multiprofissional no manejo a esses pacientes, em especial, os enfermeiros.

A descrição das enfermeiras sobre experiências quanto ao cuidado com estomias foi direcionada à troca do equipamento coletor de efluente. O cuidado à pessoa com estomia de eliminação é centrado no equilíbrio entre avaliação 
da pele periestomia e estomia, funcionalidade do estoma, adequação do equipamento coletor, fornecimento de orientações e apoio psicossocial. A partir dessa compreensão, é fundamental que o enfermeiro possua domínio sobre esses pressupostos, executando, em sua prática assistencial, a avaliação integral do indivíduo ${ }^{(2,17-18)}$, e não apenas centrar-se na troca do equipamento coletor.

Com relação aos desafios enfrentados pelas participantes no trabalho, questões referentes ao domínio do conteúdo, disponibilidade de materiais e equipamentos, rotina do serviço e trabalho colaborativo foram evidenciadas. Pesquisas que discutiram os desafios, para a profissão de enfermagem, discorreram sobre apontamentos semelhantes ao citado pelas participantes deste estudo, sendo destacados os desafios quanto à valorização profissional, falta de materiais e equipamentos, relacionamento interpessoal, recursos humanos insuficientes e sentimento de desvalia no ambiente de trabalho ${ }^{(19-20)}$. Esses achados consistem em sinais que evidenciam esgotamento da enfermagem no que diz respeito às demandas da assistência, o que destaca a necessidade em debater esses resultados e investir em melhora nas condições de trabalho ${ }^{(20-21)}$.

Percebeu-se, ao longo das discussões, imprecisão a respeito de evidências técnicas e protocolos clínicos adotados no cuidado a pessoas com estomas de eliminação. A existência de lacunas no conhecimento sobre esses estomas desde a formação profissional e dificuldades, para a promoção de atualizações profissionais que abordem o tema, contribuem para o déficit de conhecimento de enfermeiros sobre cuidados com estomias $^{(4,22)}$. A EPS apresenta-se, então, como um recurso que permite estabelecer uma associação entre a realidade vivenciada pelos enfermeiros e evidências científicas, resultando na formação de novos conhecimentos, com base na educação participativa, com consequente aprimoramento das práticas profissionais ${ }^{(8)}$.

Pesquisas comprovam que essa ferramenta é eficaz, porque envolve o desenvolvimento da aprendizagem significativa, o que pressupõe que novos conhecimentos poderão ser adquiridos desde que o indivíduo esteja disposto a aprender e se o conteúdo considerar experiências e vivências prévia dos sujeitos. Essa combinação de conhecimento novo com aqueles já existentes permite que o profissional de saúde elabore novos significados e conceitos à sua prática clínica ${ }^{(23-24)}$.
Investir em ações de educação permanente requer planejamento quanto aos métodos que podem ser utilizados e o público a ser abordado. Nesse aspecto, a técnica de grupo focal representa alternativa dinâmica que envolve a interação de ideias, a problematização baseada na realidade local e a construção de conhecimentos individuais e coletivos ${ }^{(25)}$.

A receptividade das participantes sobre a técnica de grupo focal demonstra a importância de se investir em métodos de reciclagem no serviço, que abordem temas presentes na dispensação da assistência de enfermagem e permitam a aquisição de novos saberes, o aperfeiçoamento de conceitos já incorporados à rotina de trabalho e à troca de experiências ${ }^{(9,25)}$.

Desse modo, ressalta-se a importância no investimento, em ambientes compatíveis com a aprendizagem profissional pelas instituições de saúde, envolvendo ações voltadas ao processo formativo das equipes de saúde, assim como o interesse dos profissionais em melhorar sua formação (26-28).

\section{CONSIDERAÇÕES FINAIS}

A educação permanente contribuiu para evidenciar as noções das participantes acerca das estomias de eliminação, evidenciou a importância do conhecimento técnico e científico para a assistência de enfermagem de qualidade e promoveu reflexões entre as participantes.

A pesquisa revelou que a capacitação profissional, utilizando a técnica de grupo focal, favorece a aquisição de conhecimento, pois permite discussões e debates que podem melhorar a prática profissional e a qualidade dos serviços de saúde, além de incentivar o compartilhamento de experiência.

Esta pesquisa contribui para a divulgação da educação permanente em saúde com tema em estomias de eliminação e da técnica de grupo focal e evidencia a importância do investimento na atualização profissional dos enfermeiros.

A pesquisa teve como limitação importante o tamanho final da amostra quanto ao número de participantes na primeira etapa, fato que interferiu nas percepções finais do estudo. Houve baixa adesão dos enfermeiros, em relação à participação na educação permanente, fato que pode estar relacionado ao horário das reuniões do grupo focal, local de realização dos encontros, ou mesmo pouco interesse em relação à temática. 


\section{REFERÊNCIAS}

1 - Vera SO, Sousa GN, Araújo SNM, Moreira WC, Damasceno CKCS, Andrade EMLR. Sexualidade de pacientes com estomias intestinais de eliminação. Rev Pesqui. 2017 [citado em 22 nov 2020]; 9(2):495-502. Acesso em: http://www.seer.unirio.br/index.php/cuidadofund amental/article/view/5451/pdf 1

2 - Lira JAC, Bezerra SMG, Oliveira AC, Rocha DM, Silva JS, Nogueira LT. Custos de equipamentos coletores e adjuvantes em pacientes com estomias de eliminação. Rev Min Enferm. 2019;23:e-1163. DOI: $10.5935 / 1415-2762.20190011$

3 - Gonçalves FGA, Reis FLM, Silva NAB, Souza NVDO, Varella TCMML, Pires AS. Conteúdo de estomaterapia e estratégias de ensino no currículo de graduação em enfermagem. Rev Enferm UERJ 2018;26:1-6. DOI: 10.12957/reuerj.2018.28921

4 - Oliveira ACM, Barros FLS, Costa AWS, Azevedo AP, Coelho PGP, Cunha MLS, et al. Conhecimento sobre o manejo de estomias intestinais de eliminação. Rev Enferm UFPE on Line 2019;13(5):1345-53. DOI: 10.5205/1981-8963v13i05a238543p1345-1353-2019

5 - Dantas FG, Souza AJG, Melo GSM, Freitas LS, Lucena SKP, Costa IKF. Prevalência de complicações em pessoas com estomias urinárias e intestinais. Revista Enfermagem Atual InDerme 2017;82(20):55-61. DOI: 10.31011/reaid-2017v.82-n.20-art.304

6 - Mello AL, Brito LJS, Terra MG, Camelo SH. Organizational strategy for the development of nurses' competences: Possibilities of Continuing Education in Health. Esc Anna Nery 2018; 22(1):e20170192. DOI: 10.1590/2177-9465-EAN2017-0192

7 - Sade PMC, Peres AM. Desenvolvimento de competências gerenciais do enfermeiro: Diretriz para serviços de educação permanente. Rev Esc Enferm USP 2015;49(6):991-8. DOI: $\underline{10.1590 / 50080-623420150000600016}$

8 - Moraes JT, Silva AE, Gontijo TL, Ribeiro RF, Faria RGS. Avaliação do impacto da capacitação no trabalho para o cuidado de pessoas com estomias. Enferm Foco 2019 [citado em 16 nov 2020]; 10(3):93-8. Acesso em: http://revista.cofen.gov.br/index.php/enfermage $\mathrm{m} /$ article/view/1810/575
9 - Ressel LB, Beck CLC, Gualda DMR, Hoffmann IC, Silva RM, Sehnem GD. O uso do grupo focal em pesquisa qualitativa. Texto Contexto Enferm. 2008; 17(4):779-86. DOI: 10.1590/S0104$\underline{07072008000400021}$

10 - Braun V, Clark V. Using thematic analysis in psychology. Qual Res Psychol. 2006;3(2):77-101. DOI: 10.1191/1478088706qp0630a

11 - Araujo MAN, Lunardi Filho WD, Alvarenga MRM, Oliveira RD, Souza JC, Vidmantas S. Perfil sociodemográfico dos enfermeiros da rede hospitalar. Rev Enferm UFPE on Line 2017;11(supl 11):4716-25. DOI: 10.5205/reuol.11138-99362-1SM.1111sup201723

12 - Santos RM, Barros LMC, Santos SA, Santos WB, Costa LMC. La inserción masculina en la Enfermería: ¿qué se ha escrito sobre esta cuestión? Cult Cuid Enferm. 2017;48(2):219-32. DOI: $10.14198 /$ cuid.2017.48

13 - Almeida EJ, Silva AL. Characterization of the epidemiological profile of the ostomy in hospitals of the secretary of state of health of the Federal District. Estima 2015;13(1):11-6. DOI: 10.5327/Z1806-3144201500010004

14 - Carlsson E, Fingren J, Hallén AM, Petersén C, Lindholm $E$. The prevalence of ostomy-related complications 1 year after ostomy surgery: A prospective, descriptive, clinical study. Osto Wou Manage. 2016 [citado em 3 nov 2020]; 62(10):34$48 . \quad$ Acesso em: https://www.ncbi.nlm.nih.gov/pubmed/27768579

15 - Taneja C, Netsch D, Rolstad BS, Inglese G, Lamerato L, Oster G. Clinical and economic burden of peristomal skin complications in patients with recent ostomies. J Wound Ostomy Continence Nurs. 2017 [citado em 3 nov 2020]; 44(4): 350-7, $2017 . \quad$ Acesso em: https://www.ncbi.nlm.nih.gov/pubmed/28574928

16 - Schreiber ML. Ostomies: nursing care and management. Medsurg Nurs. 2016 [citado em 15 out 2020]; 25(2):127-30. Acesso em: https://www.ncbi.nlm.nih.gov/pubmed/27323475

17 - Coqueiro JM, P Rodrigues ASSJ, Figueiredo TAM. A produção do cuidado ao usuário estomizado: Considerações da equipe de enfermagem. Rev Enferm UFPE on Line 
2015;9(6):8148-54. DOI: $10.5205 /$ reuol.758566362-1-ED.0906201501

18 - Maurício VC, Souza NVDO, Costa CCP, Dias MO. The view of nurses about educational practices targeted at people with a stoma. Esc Anna Nery 2017;21(4):e20170003. DOI: 10.1590/2177-9465-ean-2017-0003

19 - Costa CCP, Soares SSS, Vieira MLC, Oliveira MD, Pedro RS, Chaves USB, et al. Stomatherapists in the world of work: Practicalities and difficulties for the professional practice. Esc Anna Nery 2021;25(2):e20200262. DOI: 10.1590/2177-9465EAN-2020-0262

20 - Silva MCN, Machado MH. Health and Work System: Challenges for the Nursing in Brazil. Ciênc Saúde Coletiva 2020;25(1):7-13. DOI: $\underline{10.1590 / 1413-81232020251.27572019}$

21 - Machado MH. Pesquisa Perfil da enfermagem no Brasil: Relatório final. Rio de Janeiro: NerhusDaps-Ensp/Fiocruz; 2017.

22 - Oliveira ACM, Barros FLS, Costa AWS et al. Knowledge about the management of intestinal stomas of elimination. J Nus UFPE on Line 2019; 13(5):1345-53. DOI: 10.5205/1981-8963$\underline{\text { v13i05a238543p1345-1353-2019 }}$

23 - Cardoso MLM, Costa PP, Costa DM, Xavier C, Souza RMP. The National Permanent Health Education Policy in Public Health Schools: Reflections from practice. Ciênc Saúde Coletiva. 2017;22(5):1489-500. DOI: 10.1590/1413$\underline{81232017225.33222016}$

24 - Fernandes FC, Cortez EA, Laprovita D, Almeida $L P$, Ferreira AF, Corvino MPF. Educação permanente em saúde sob a perspectiva de Agostinho de Hipona. Rev Bras Enferm. 2017; 70(3):656-61. DOI: 10.1590/0034-7167-2016-0484

25 - Kinalski DDF, Paula CC, Padoin SMM, Neves ET, Kleinubing RE, Cortes LF. Focus group on qualitative research: Experience report. Rev Bras Enferm. 2017;70(2):424-9. DOI: 10.1590/00347167-2016-0091

26 - Lavich CRP, Terra MG, Mello AL, Raddatz M, Arnemann CT. Ações de educação permanente dos enfermeiros facilitadores de um núcleo de educação em enfermagem. Rev Gaúcha Enferm. 2017;38(1):e62261. DOI: $10.1590 / 1983-$ 1447.2017.01.62261
27 - Sá ANMGN, Ferreira ERO, Xavier JC, Alves CM. Contribuições da educação permanente para qualificação da assistência de enfermagem em um hospital público. Rev Bras Ciênc Saúde 2018;22(1): 87-94. DOI: 10.4034/RBCS.2018.22.01.1

28 - Nogueira IS, Acioli S, Carreira L, Baldissera VDA. Older adult care: Permanent education practices of the Family Health Support Center. Rev Esc Enferm USP 2019;53:e03512. DOI: $\underline{10.1590 / S 1980-220 \times 2018022103512}$

Nota: Artigo extraído da pesquisa intitulada "O Cuidado de Enfermagem a Pessoa Com Estomia de Eliminação", vinculada a Universidade Federal de Mato Grosso/CUA.

Recebido em: 05/12/2020

Aprovado em: 07/04/2021 